\title{
Applications of Machine Vision in Flight Test
}

\author{
Phil M. Westhart, Hakima Ibaroudene, Austin J. Whittington, Myron L. Moodie, Ben A. Abbott, Ph.D. \\ Southwest Research Institute ${ }^{\circledR}\left(S w R /{ }^{\circledR}\right)$, San Antonio, TX, USA \\ fti@swri.org
}

\begin{abstract}
:
Machine vision uses cameras, computers, and algorithms to replace human vision in various applications such as factory automation, autonomous vehicles, object recognition, optical character recognition, and many other applications. Existing machine vision technologies can be applied to the flight test domain to solve real world problems, such as extracting data from cockpit displays in realtime. These technologies can reduce installation costs and complexity and may not require aircraft modification to obtain parameter data. This paper explores machine vision techniques for flight test applications and includes a case study of a portable cockpit display video measurement system that reads digital measurements from aircraft instrumentation panels.
\end{abstract}

Key words: Machine vision, cockpit display, video measurement

\section{Introduction}

Machine vision has been leveraged in a variety of domains with many innovative applications. SwRI has been actively performing research in the field of machine vision for over two decades. Some recent machine vision applications include a machine-learning based detection of hazardous chemical spills [1], package sorting in distribution warehouses [2], and enabling autonomous vehicles in GPS degraded environments [3]. Recent efforts have brought this machine vision expertise to approach challenges in the flight test community, specifically targeting increased efficiency in test article preparation.

Due to the increasing complexity of airframes and associated controls for modern aircraft, the test instrumentation is required to be similarly complex, which requires significant investments in manpower and material to prepare an aircraft for test. The developmental flight test of modern commercial aircraft typically requires eight months to a year of test time, in addition to three to six months of planning and installation of instrumentation systems in various locations in the aircraft. While the flight test tools have evolved over time, a constant theme we have observed with all our customers is the need to reduce time and cost required for a test.

\section{Machine Vision Challenges}

Flight test environments introduce inherent challenges to traditional machine vision techniques, most notably high vibration and lighting variation that are driven by the dynamic operating environment of the aircraft. In addition, cost and weight minimization typically requires a single flight test camera covering as large a region as practical which leads to low resolution of features. When flight test cameras are used to capture other active displays such as glass cockpit displays, the unsynchronized frame rates of the displays and cameras produces aliasing and interleaving effects. Traditional approaches must be modified to accommodate for these difficulties.

Since the camera vibrates independently of the observed item, noise is introduced into recorded and live video streams which can negatively affect data extraction. To correct for these errors, landmarks can be identified through calibration and automatically tracked and shifted to ensure they are in the same location. After initial stabilization, vibration is further mitigated by looking at aggregate data across multiple frames of the video feed. This increases the stability of the features to be measured, resulting in more reliable images for data extraction.

While standard machine vision techniques have a variety of feature recognition capabilities, the flight test environment impacts their performance. For example, the performance of standard optical character recognition (OCR) libraries are significantly linked to particular font sets. These approaches often cannot handle font distortions introduced in a flight test environment such as adverse lighting conditions, obliquities in font representation, 
and artifacts from interleaved video compression.

We have been able to overcome these limitations by augmenting the machine vision techniques with a combination of calibrationdriven image processing techniques. In general, all of the calibration approaches use a form of "template." That is, imagery that has been collected directly from the display of interest will be used to augment the vision process. This calibration information can then allow the system to "learn" and adapt to the particular setup. For example, the specific distortion to apply is calculated during an initial calibration step and adapted and applied to subsequent data extractions to be able to achieve the necessary measurement accuracy.

\section{Case Study: Cockpit Display Video Measurement}

Typically, flight test measurements are acquired through a combination of adding discrete aeromechanical sensors to the structure and tapping into the communication busses between flight control computers. The amount and type of measurement sources depends on a combination of the maturity of the aircraft design under test and what specific test objectives need to be achieved. Historically, smaller test programs (such as testing a modification to an aircraft design that has already been certified in a prior large test program) have simply used a subset of the same instrumentation used for the most involved large test programs. While this minimizes purchasing multiple types of instrumentation equipment, it means that installation effort cannot drop below the minimum required by a subset of that instrumentation technology. Recent industry efforts have focused on leveraging wireless sensing components to remove the cost (install time, cost, and weight) of cabling, but the results have been hampered by practical considerations of power management and batteries.

The growth of storage capacity for flight test data combined with falling costs of video cameras driven by other industries has led to a significant increase in the amount of video acquired from cockpit displays, control surfaces, and actuators. While many other industries have leveraged video cameras with machine vision techniques to monitor and control processes, the flight test industry has largely used the various video sources either as a secondary data source that is only inspected manually when measurements from other sensors conflict, or by manually acquiring measurements from the video through human inspection.

Seeing this landscape, we have begun to socialize a different strategy to help achieve the goal of reduced time and cost of flight test. Specifically, we feel that if measurement information can be reliably automatically processed from flight test cameras observing cockpit displays, then this instrumentation could potentially reduce or eliminate the need for more traditional instrumentation systems in certain low-instrumentation flight test programs such as supplemental type certificate (STC) or production test.

We have developed a prototype concept demonstration that detects a limited set of numeric display information from cockpit video and begun a dialog with various flight test programs to uncover use cases and requirements which will influence our further research and development. While these demos are far from complete implementations, the results achieved so far and the feedback we have received make us hopeful that a finalized system can provide an effective capability to flight test users.

We have compensated for the constraints of the flight test use case by augmenting traditional machine vision techniques with test-setup specific calibration and additional image processing approaches not typically needed in more controlled machine vision environments. This idea, while a natural fit for the flight test domain, is not common in other industries. Flight test, on the other hand, is comfortable with detailed calibration procedures. We expect that requiring a calibration procedure per install would require less overall setup time than using traditional instrumentation such that the goal of reduced time and cost required for a test is still achieved.

Using the machine vision techniques we have developed, data has been successfully extracted from flight test video of a helicopter's avionics display. These test case videos were from typical flight test operations with a combination of impairments from vibration, lighting, and momentary blockage by pilot movement. The software requires a video stream that captures the avionics system display, but it is forgiving of camera positioning up to 30 degrees off normal. This video can be either acquired and processed in real-time on a test aircraft or lab bench avionics display, or post-processed from a recorded video file.

The setup calibration approach requires the user to point the camera at the gauge from a distance equivalent to the back of the cockpit 
and then mark several features and regions of interest in the image of the gauge. The ability for the algorithms to continually extract measurements without intervention after calibration provides validation of the techniques. Results from the helicopter flight test video extracted using this system have shown $>99 \%$ fidelity when compared to truth data acquired from available data busses. The results can be seen in Figure 1.

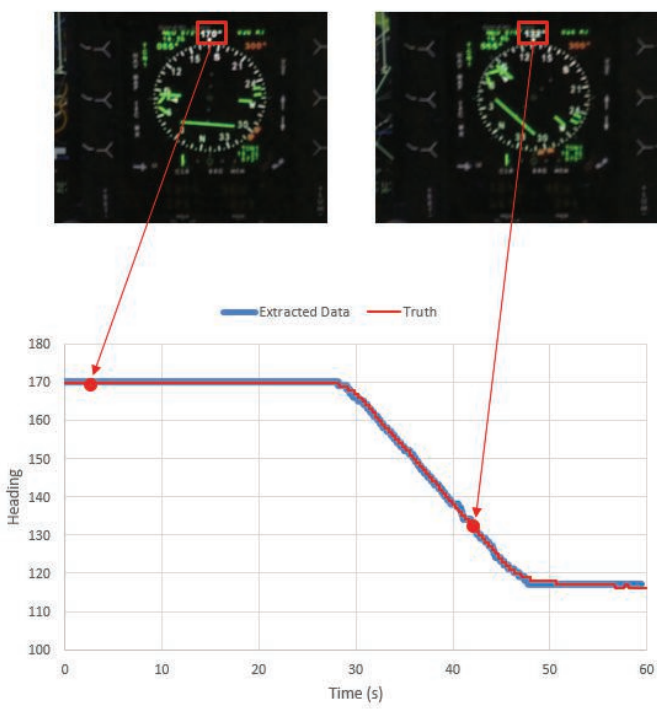

Fig. 1. Measurements extracted from video processing compared to truth data (video courtesy Airbus Helicopters).

The machine vision techniques described here provide a less expensive alternative for gathering and extracting data during a flight test. Traditional methods involve tapping an avionics bus for existing instrumentation, adding the weight and complexity of a bus monitor that must have physical access to the test article's internals.

Machine vision techniques, used to extract data from cockpit displays, provide a bridge between these techniques. The avionics bus data can be reproduced, based on observations of the displays, without requiring the invasive installation of a bus monitor. Detailed knowledge of the bus catalog of the test article, what messages correspond to what values, and in what units, is no longer needed, as engineers can intuitively extract the values of note by associating measurements with the corresponding instrument. The flexibility of the camera is still preserved, as multiple passes can be made post-flight to extract data that had no prior use, but suddenly was relevant in postprocessing.
Building on the initial prototype capabilities, we envision the system will ultimately have the capabilities to extract data from a variety of cockpit display types including:

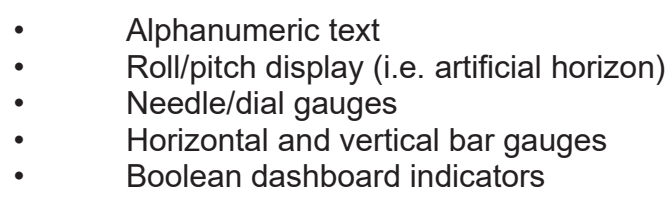

This set of displays covers the set of traditional aircraft instrumentation, including both the instruments for visual flight rules and instrument flight rules. It is also capable of monitoring status of many more advanced, specific instruments presenting data matching these categories and can be customized to specific flight program needs.

Like all measurement systems, consistent time marking is essential. IEEE 1588 PTP time of each acquired video frame is tracked and timestamps are applied to each measurement instance. The system supports adjusting the timestamps by an offset to compensate for video display and camera delays if needed. This provides the data with the timing accuracy necessary for comparative processing with other measurement sources.

Additionally, by utilizing portable and modular software techniques rather than hardwarespecific processing, we are able to adapt to a wide variety of platforms from small embedded systems to general purpose PCs. Both the optical and platform components are independent from the software since the calibration process normalizes variations.

In addition to data extraction from avionics displays we see potential in measuring a variety of physical phenomena using similar specialized applications of machine vision. Possibilities include measuring wing deflection, flutter and vibration analysis, ice accumulation measurement, and engine analysis. Expensive and time-consuming installation of sensors could be replaced by the simple installation of a camera, where the nature of the physical event to be measured and the resolution of the camera allow.

\section{Conclusion}

This paper has provided a discussion of the challenges associated with using machine vision techniques in a flight test environment. We have had success in enhancing traditional machine vision techniques in order to overcome these challenges, resulting in a solution that has the potential to provide flight test users a rapid-deployment data acquisition capability. 


\section{References}

[1] SwRI Smart Leak Detection System Locates Hazardous Chemical Spills,

https://www.swri.org/press-release/swri-smartleak-detection-system-locates-hazardouschemical-spills

[2] Blazing a Trail For Smarter, More Agile, Automation,

https://www.swri.org/sites/default/files/publication s/technology-today-summer-2017.pdf, p4-9

[3] SwRl's Ranger Localization Technology Allows Precise Automated Driving,

https://www.swri.org/press-

release/swri\%E2\%80\%99s-ranger-localizationtechnology-allows-precise-automated-driving 